\title{
Uniqueness and existence of positive solutions for a multi-point boundary value problem of singular fractional differential equations
}

\author{
Wen-Xue Zhou ${ }^{1,2^{*}}$, Yan-Dong Chu ${ }^{2}$ and Dumitru Băleanu $u^{3,4}$
}

\section{"Correspondence:}

wxzhou2006@126.com

'School of Mathematical Sciences,

Fudan University, Shanghai, 200433,

China

${ }^{2}$ Department of Mathematics, Lanzhou Jiaotong University, Lanzhou, 730070, China

Full list of author information is available at the end of the article

\begin{abstract}
In this paper, we study the uniqueness of a positive solution for the singular nonlinear fractional differential equation boundary value problem $D_{0+}^{\alpha} u(t)+f(t, u(t))=0$,

$0<t<1, u(0)=0, u(1)=\left.a D^{\frac{\alpha-1}{2}} u(t)\right|_{t=\xi}$, where $1<\alpha \leq 2$ is a real number, $D_{0+}^{\alpha}$ is the standard Riemann-Liouville differentiation and $f:(0,1] \times[0,+\infty) \rightarrow[0,+\infty)$, with $\lim _{t \rightarrow 0^{+}} f(t, \cdot)=+\infty$. Our analysis relies on a fixed-point theorem in partially ordered set. As an application, an example is presented to illustrate the main result.

MSC: 26A33; 34B15; 34K37
\end{abstract}

Keywords: boundary value problem; singular fractional differential equations; Riemann-Liouville fractional derivative; uniqueness; partially ordered set

\section{Introduction}

Fractional differential equations have gained considerable importance due to their application in various sciences, such as physics, mechanics, chemistry, engineering, etc. In recent years, there has been a significant development in ordinary and partial differential equations involving fractional derivatives; see the monographs of Kilbas et al. [1], Miller and Ross [2], Oldham and Spanier [3], Podlubny [4], Samko [5], and the papers [6-16] and the references therein.

However, there are few papers, which have considered the singular boundary value problems of fractional differential equations; see [17-23]. In particular, Delbosco and Rodino [17] considered the existence of a solution for the nonlinear fractional differential equation $D_{0+}^{\alpha} u=f(t, u)$, where $0<\alpha<1$ and $f:[0, a] \times \mathbb{R} \rightarrow \mathbb{R}, 0<a \leq+\infty$ is a given continuous function in $(0, a) \times \mathbb{R}$. They obtained some results for solutions by using the Schauder fixed-point theorem and the Banach contraction principle.

Qiu and Bai [18] considered the existence of a positive solution to boundary value problems of the nonlinear fractional differential equation

$$
\begin{aligned}
& { }^{c} D_{0+}^{\alpha} u(t)+f(t, u(t))=0, \quad 0<t<1,2<\alpha \leq 3, \\
& u(0)=u^{\prime}(1)=u^{\prime \prime}(0)=0,
\end{aligned}
$$

๑ 2013 Zhou et al.; licensee Springer. This is an Open Access article distributed under the terms of the Creative Commons Attribution License (http://creativecommons.org/licenses/by/2.0), which permits unrestricted use, distribution, and reproduction in any medium, provided the original work is properly cited. 
where ${ }^{c} D_{0+}^{\alpha}$ is the Caputo fractional derivative, and $f:(0,1] \times[0,+\infty) \rightarrow[0,+\infty)$, with $\lim _{t \rightarrow 0^{+}} f(t, \cdot)=+\infty$ (i.e., $f$ is singular at $t=0$ ). They obtained the existence of positive solutions by means of the Guo-Krasnosel'skii fixed-point theorem and nonlinear alternative of Leray-Schauder type in a cone. In [18], the uniqueness of the solution is not treated.

From the above works, we can see a fact, although the fractional boundary value problems have been investigated by some authors, to the best of our knowledge, there have been few papers which deal with the problem (1.1)-(1.2) for nonlinear singular fractional differential equation. Motivated by all the works above, this paper is mainly concerned with the uniqueness of a positive solution for the singular nonlinear fractional differential equation boundary value problem

$$
\begin{aligned}
& D_{0^{+}}^{\alpha} u(t)+f(t, u(t))=0, \quad 0<t<1, \\
& u(0)=0, \quad u(1)=\left.a D^{\frac{\alpha-1}{2}} u(t)\right|_{t=\xi},
\end{aligned}
$$

where $1<\alpha \leq 2$ is a real number, $\xi \in\left(0, \frac{1}{2}\right], a \in(0,+\infty)$ satisfy $a \Gamma(\alpha) \xi^{\frac{\alpha-1}{2}}<\Gamma\left(\frac{\alpha+1}{2}\right)$, and $D_{0^{+}}^{\alpha}$ is the standard Riemann-Liouville differentiation, and $f:(0,1] \times[0,+\infty) \rightarrow[0,+\infty)$, with $\lim _{t \rightarrow 0^{+}} f(t, \cdot)=+\infty$. In this article, by using a fixed- point theorem in partially ordered set, existence and uniqueness results of a positive solution for the problem (1.1)-(1.2) are given.

The paper is organized as follows. In Section 2, we give some preliminary results that will be used in the proof of the main results. In Section 3, we establish the uniqueness of a positive solution for the singular nonlinear fractional differential equation boundary value problem (1.1)-(1.2). In the end, we illustrate a simple use of the main result.

\section{Preliminaries and lemmas}

For the convenience of the reader, we present here the necessary definitions from fractional calculus theory. These definitions can be found in the recent literature such as [1] and $[4]$.

Definition 2.1 $[1,4]$ The Riemann-Liouville fractional integral of order $\alpha>0$ of a function $f:(0,+\infty) \rightarrow \mathbb{R}$ is given by

$$
I_{0^{+}}^{\alpha} f(t)=\int_{0}^{t} \frac{(t-s)^{\alpha-1}}{\Gamma(\alpha)} f(s) d s
$$

provided that the right side is pointwise defined on $(0,+\infty)$, where $\Gamma$ is the gamma function.

Definition 2.2 [1, 4] The Riemann-Liouville fractional derivative of order $\alpha>0$ of a continuous function $f:(0,+\infty) \rightarrow \mathbb{R}$ is given by

$$
\left(D_{0+}^{\alpha} f\right)(t)=\frac{1}{\Gamma(n-\alpha)}\left(\frac{d}{d t}\right)^{n} \int_{0}^{t}(t-s)^{n-\alpha+1} f(s) d s,
$$

provided that the right side is pointwise defined on $(0,+\infty)$. Here, $n=[\alpha]+1$ and $[\alpha]$ denotes the integer part of $\alpha$. 
Lemma 2.1 [1] Let $\alpha>0$. If we assume $u \in C(0,1) \cap L(0,1)$, then the fractional differential equation

$$
D_{0+}^{\alpha} u(t)=0
$$

has

$$
u(t)=C_{1} t^{\alpha-1}+C_{2} t^{\alpha-2}+\cdots+C_{N} t^{\alpha-N}, \quad C_{i} \in \mathbb{R}, i=1,2, \ldots, N,
$$

as unique solutions, where $N$ is the smallest integer greater than or equal to $\alpha$.

Lemma 2.2 [1] Assume that $h \in C(0,1) \cap L(0,1)$ with a fractional derivative of order $\alpha>0$ that belongs to $C(0,1) \cap L(0,1)$. Then

$$
I_{0+}^{\alpha} D_{0+}^{\alpha} h(t)=h(t)+C_{1} t^{\alpha-1}+C_{2} t^{\alpha-2}+\cdots+C_{N} t^{\alpha-N},
$$

for some $C_{i} \in \mathbb{R}, i=1,2, \ldots, N$, where $N$ is the smallest integer greater than or equal to $\alpha$.

Lemma 2.3 [21] Let $h \in C(0,1) \cap L(0,1)$ and $1<\alpha \leq 2$, $\xi \in(0,1), a \in \mathbb{R}$ satisfy that $a \Gamma(\alpha) \xi^{\frac{\alpha-1}{2}} \neq \Gamma\left(\frac{\alpha+1}{2}\right)$, then the unique solution of

$$
\begin{aligned}
& D_{0+}^{\alpha} u(t)+h(t)=0, \quad 0<t<1, \\
& u(0)=0, \quad u(1)=\left.a D^{\frac{\alpha-1}{2}} u(t)\right|_{t=\xi}
\end{aligned}
$$

is given by

$$
\begin{aligned}
u(t)= & -\int_{0}^{t} \frac{(t-s)^{\alpha-1}}{\Gamma(\alpha)} h(s) d s \\
& +\frac{t^{\alpha-1} \Gamma\left(\frac{\alpha+1}{2}\right)}{\Gamma\left(\frac{\alpha+1}{2}\right)-a \Gamma(\alpha) \xi^{\frac{\alpha-1}{2}}}\left\{\int_{0}^{1} \frac{(1-s)^{\alpha-1}}{\Gamma(\alpha)} h(s) d s-a \int_{0}^{\xi} \frac{(\xi-s)^{\frac{\alpha-1}{2}}}{\Gamma\left(\frac{\alpha+1}{2}\right)} h(s) d s\right\} .
\end{aligned}
$$

Lemma 2.4 [21] Let $h \in C((0,1),[0,+\infty)) \cap L(0,1)$ and $1<\alpha \leq 2, \xi \in\left(0, \frac{1}{2}\right], a \in(0,+\infty)$ satisfy that $a \Gamma(\alpha) \xi^{\frac{\alpha-1}{2}}<\Gamma\left(\frac{\alpha+1}{2}\right)$, then the unique solution of the problem (2.1)-(2.2)

$$
\begin{aligned}
u(t)= & -\int_{0}^{t} \frac{(t-s)^{\alpha-1}}{\Gamma(\alpha)} h(s) d s \\
& +\frac{t^{\alpha-1} \Gamma\left(\frac{\alpha+1}{2}\right)}{\Gamma\left(\frac{\alpha+1}{2}\right)-a \Gamma(\alpha) \xi^{\frac{\alpha-1}{2}}}\left\{\int_{0}^{1} \frac{(1-s)^{\alpha-1}}{\Gamma(\alpha)} h(s) d s-a \int_{0}^{\xi} \frac{(\xi-s)^{\frac{\alpha-1}{2}}}{\Gamma\left(\frac{\alpha+1}{2}\right)} h(s) d s\right\} \\
= & \int_{0}^{1} G(t, s) h(s) d s+\frac{a t^{\alpha-1}}{\Gamma\left(\frac{\alpha+1}{2}\right)-a \Gamma(\alpha) \xi^{\frac{\alpha-1}{2}}} \\
& \times\left\{\int_{0}^{\xi}\left[(1-s)^{\alpha-1} \xi^{\frac{\alpha-1}{2}}-(\xi-s)^{\frac{\alpha-1}{2}}\right] h(s) d s+\int_{\xi}^{1}(1-s)^{\alpha-1} \xi^{\frac{\alpha-1}{2}} h(s) d s\right\}
\end{aligned}
$$


is nonnegative on $[0,1]$, where

$$
G(t, s)= \begin{cases}\frac{[t(1-s)]^{q-1}-(t-s)^{q-1}}{\Gamma(q)}, & \text { if } 0 \leq s \leq t \leq 1, \\ \frac{[t(1-s)]^{q-1}}{\Gamma(q)}, & \text { if } 0 \leq t \leq s \leq 1 .\end{cases}
$$

The following two lemmas are fundamental in the proofs of our main result.

Lemma $2.5[24]$ Let $(E, \leq)$ be a partially ordered set and suppose that there exists a metric $d$ in E such that $(E, d)$ is a complete metric space. Assume that E satisfies:

If $x_{n}$ is a nondecreasing sequence in E such that $x_{n} \rightarrow x$ then $x_{n} \leq x, \quad \forall n \in \mathbb{N}$.

Let $f: E \rightarrow E$ be a nondecreasing mapping such that

$$
d(f(x), f(y)) \leq d(x, y)-\varphi(d(x, y)), \quad \text { for } x \geq y
$$

where $\varphi:[0,+\infty) \rightarrow[0,+\infty)$ is continuous and nondecreasing function such that $\varphi$ is positive in $(0,+\infty), \varphi(0)=0$ and $\lim _{t \rightarrow+\infty} \varphi(t)=+\infty$. If there exists $x_{0} \in E$ with $x_{0} \leq f\left(x_{0}\right)$, then $f$ has a fixed point.

If we consider that $(E, \leq)$ satisfies the following condition:

For $x, y \in E$ there exists $z \in E$ which is comparable to $x$ and $y$,

then we have the following result.

Lemma 2.6 [24] Adding condition (2.7) to the hypotheses of Lemma 2.5, we obtain uniqueness of the fixed point off.

\section{Main results}

Theorem 3.1 Let $1<\alpha \leq 2, \xi \in\left(0, \frac{1}{2}\right], a \in(0,+\infty)$ satisfy that $a \Gamma(\alpha) \xi^{\frac{\alpha-1}{2}}<\Gamma\left(\frac{\alpha+1}{2}\right), F$ : $(0,1] \rightarrow[0,+\infty)$ is continuous, and $\lim _{t \rightarrow 0^{+}} F(t)=+\infty$. Suppose that there exists a constant $\sigma: 0<\sigma<1$ such that $t^{\sigma} F(t)$ is a continuous function on $[0,1]$. Then the unique solution of the problem (2.1)-(2.2) is given by

$$
\begin{aligned}
H(t)= & -\int_{0}^{t} \frac{(t-s)^{\alpha-1}}{\Gamma(\alpha)} F(s) d s \\
& +\frac{t^{\alpha-1} \Gamma\left(\frac{\alpha+1}{2}\right)}{\Gamma\left(\frac{\alpha+1}{2}\right)-a \Gamma(\alpha) \xi^{\frac{\alpha-1}{2}}}\left\{\int_{0}^{1} \frac{(1-s)^{\alpha-1}}{\Gamma(\alpha)} F(s) d s-a \int_{0}^{\xi} \frac{(\xi-s)^{\frac{\alpha-1}{2}}}{\Gamma\left(\frac{\alpha+1}{2}\right)} F(s) d s\right\} \\
= & \int_{0}^{1} G(t, s) F(s) d s+\frac{a t^{\alpha-1}}{\Gamma\left(\frac{\alpha+1}{2}\right)-a \Gamma(\alpha) \xi^{\frac{\alpha-1}{2}}} \\
& \times\left\{\int_{0}^{\xi}\left[(1-s)^{\alpha-1} \xi^{\frac{\alpha-1}{2}}-(\xi-s)^{\frac{\alpha-1}{2}}\right] F(s) d s+\int_{\xi}^{1}(1-s)^{\alpha-1} \xi^{\frac{\alpha-1}{2}} F(s) d s\right\}
\end{aligned}
$$

and is continuous on $[0,1]$. 
Proof By the continuity of $t^{\sigma} F(t)$, it is easy to check that $H(0)=0$. The proof is divided into three cases.

Case 1. $t_{0}=0, \forall t \in(0,1]$.

Since $t^{\sigma} F(t)$ is continuous in $[0,1]$, there exists a constant $M>0$, such that $\left|t^{\sigma} F(t)\right| \leq M$, $t \in[0,1]$. Hence,

$$
\begin{aligned}
& |H(t)-H(0)| \\
& =\mid \int_{0}^{1} G(t, s) F(s) d s+\frac{a t^{\alpha-1}}{\Gamma\left(\frac{\alpha+1}{2}\right)-a \Gamma(\alpha) \xi^{\frac{\alpha-1}{2}}} \\
& \times\left\{\int_{0}^{\xi}\left[(1-s)^{\alpha-1} \xi^{\frac{\alpha-1}{2}}-(\xi-s)^{\frac{\alpha-1}{2}}\right] F(s) d s+\int_{\xi}^{1}(1-s)^{\alpha-1} \xi^{\frac{\alpha-1}{2}} F(s) d s\right\} \mid \\
& =\mid \int_{0}^{1} G(t, s) s^{-\sigma} s^{\sigma} F(s) d s+\frac{a t^{\alpha-1}}{\Gamma\left(\frac{\alpha+1}{2}\right)-a \Gamma(\alpha) \xi^{\frac{\alpha-1}{2}}} \\
& \times\left\{\int_{0}^{\xi}\left[(1-s)^{\alpha-1} \xi^{\frac{\alpha-1}{2}}-(\xi-s)^{\frac{\alpha-1}{2}}\right] s^{-\sigma} s^{\sigma} F(s) d s+\int_{\xi}^{1}(1-s)^{\alpha-1} \xi^{\frac{\alpha-1}{2}} s^{-\sigma} s^{\sigma} F(s) d s\right\} \mid \\
& \leq M \mid \int_{0}^{t}\left[\frac{[t(1-s)]^{\alpha-1}-(t-s)^{\alpha-1}}{\Gamma(\alpha)}\right] s^{-\sigma} d s+\int_{t}^{1} \frac{[t(1-s)]^{\alpha-1}}{\Gamma(\alpha)} s^{-\sigma} d s \\
& +\frac{a t^{\alpha-1}}{\Gamma\left(\frac{\alpha+1}{2}\right)-a \Gamma(\alpha) \xi^{\frac{\alpha-1}{2}}} \times\left\{\int_{0}^{\xi}\left[(1-s)^{\alpha-1} \xi^{\frac{\alpha-1}{2}}-(\xi-s)^{\frac{\alpha-1}{2}}\right] s^{-\sigma} d s\right. \\
& \left.+\int_{\xi}^{1}(1-s)^{\alpha-1} \xi^{\frac{\alpha-1}{2}} s^{-\sigma} d s\right\} \mid \\
& \leq M \frac{t^{\alpha-1}}{\Gamma(\alpha)} B(1-\sigma, \alpha)+M \frac{t^{\alpha-\sigma}}{\Gamma(\alpha)} B(1-\sigma, \alpha) \\
& +\frac{a M t^{\alpha-1} \xi^{\frac{\alpha-1}{2}}}{\Gamma\left(\frac{\alpha+1}{2}\right)-a \Gamma(\alpha) \xi^{\frac{\alpha-1}{2}}} B(1-\sigma, \alpha)+\frac{a M t^{\alpha-1} \xi^{1-\sigma+\frac{\alpha-1}{2}}}{\Gamma\left(\frac{\alpha+1}{2}\right)-a \Gamma(\alpha) \xi^{\frac{\alpha-1}{2}}} B\left(1-\sigma, \frac{\alpha+1}{2}\right) \\
& \leq M \frac{\Gamma(1-\sigma)}{\Gamma(1-\sigma+\alpha)}\left[t^{\alpha-1}+t^{\alpha-\sigma}\right] \\
& +\frac{a M t^{\alpha-1} \xi^{\frac{\alpha-1}{2}}}{\Gamma\left(\frac{\alpha+1}{2}\right)-a \Gamma(\alpha) \xi^{\frac{\alpha-1}{2}}} \frac{\Gamma(1-\sigma) \Gamma(\alpha)}{\Gamma(1-\sigma+\alpha)}+\frac{a M t^{\alpha-1} \xi^{1-\sigma+\frac{\alpha-1}{2}}}{\Gamma\left(\frac{\alpha+1}{2}\right)-a \Gamma(\alpha) \xi^{\frac{\alpha-1}{2}}} \frac{\Gamma(1-\sigma) \Gamma\left(\frac{\alpha+1}{2}\right)}{\Gamma\left(1-\sigma+\frac{\alpha+1}{2}\right)} \\
& \rightarrow 0 \quad(t \rightarrow 0) \text {, }
\end{aligned}
$$

where $B(\cdot, \cdot)$ denotes the beta function.

Case 2. $t_{0} \in(0,1), \forall t \in\left(t_{0}, 1\right]$.

$$
\begin{aligned}
\mid H(t) & -H\left(t_{0}\right) \mid \\
= & \mid \int_{0}^{1} G(t, s) s^{-\sigma} s^{\sigma} F(s) d s-\int_{0}^{1} G\left(t_{0}, s\right) s^{-\sigma} s^{\sigma} F(s) d s \\
& +\frac{a\left[t^{\alpha-1}-t_{0}^{\alpha-1}\right]}{\Gamma\left(\frac{\alpha+1}{2}\right)-a \Gamma(\alpha) \xi^{\frac{\alpha-1}{2}}} \times\left\{\int_{0}^{\xi}\left[(1-s)^{\alpha-1} \xi^{\frac{\alpha-1}{2}}-(\xi-s)^{\frac{\alpha-1}{2}}\right] s^{-\sigma} s^{\sigma} F(s) d s\right. \\
& \left.+\int_{\xi}^{1}(1-s)^{\alpha-1} \xi^{\frac{\alpha-1}{2}} s^{-\sigma} s^{\sigma} F(s) d s\right\} \mid
\end{aligned}
$$




$$
\begin{aligned}
& \leq M \mid \int_{0}^{t} \frac{[t(1-s)]^{\alpha-1}-(t-s)^{\alpha-1}}{\Gamma(\alpha)} s^{-\sigma} d s+\int_{t}^{1} \frac{[t(1-s)]^{\alpha-1}}{\Gamma(\alpha)} s^{-\sigma} d s \\
& -\int_{t_{0}}^{1} \frac{\left[t_{0}(1-s)\right]^{\alpha-1}}{\Gamma(\alpha)} s^{-\sigma} d s-\int_{0}^{t_{0}} \frac{\left[t_{0}(1-s)\right]^{\alpha-1}-\left(t_{0}-s\right)^{\alpha-1}}{\Gamma(\alpha)} s^{-\sigma} d s \\
& +\frac{a\left[t^{\alpha-1}-t_{0}^{\alpha-1}\right]}{\Gamma\left(\frac{\alpha+1}{2}\right)-a \Gamma(\alpha) \xi^{\frac{\alpha-1}{2}}} \times\left\{\int_{0}^{\xi}\left[(1-s)^{\alpha-1} \xi^{\frac{\alpha-1}{2}}-(\xi-s)^{\frac{\alpha-1}{2}}\right] s^{-\sigma} d s\right. \\
& \left.+\int_{\xi}^{1}(1-s)^{\alpha-1} \xi^{\frac{\alpha-1}{2}} s^{-\sigma} d s\right\} \mid \\
& =M \mid \frac{t^{\alpha-1}-t_{0}{ }^{\alpha-1}}{\Gamma(\alpha)} \int_{0}^{1}(1-s)^{\alpha-1} s^{-\sigma} d s+\int_{t_{0}}^{t} \frac{(t-s)^{\alpha-1}}{\Gamma(\alpha)} s^{-\sigma} d s \\
& +\int_{0}^{t_{0}} \frac{(t-s)^{\alpha-1}-\left(t_{0}-s\right)^{\alpha-1}}{\Gamma(\alpha)} s^{-\sigma} d s+\frac{a\left[t^{\alpha-1}-t_{0}^{\alpha-1}\right]}{\Gamma\left(\frac{\alpha+1}{2}\right)-a \Gamma(\alpha) \xi^{\frac{\alpha-1}{2}}} \\
& \times\left\{\int_{0}^{\xi}\left[(1-s)^{\alpha-1} \xi^{\frac{\alpha-1}{2}}-(\xi-s)^{\frac{\alpha-1}{2}}\right] s^{-\sigma} d s+\int_{\xi}^{1}(1-s)^{\alpha-1} \xi^{\frac{\alpha-1}{2}} s^{-\sigma} d s\right\} \mid \\
& \leq M\left\{\frac{t^{\alpha-1}-t_{0}^{\alpha-1}}{\Gamma(\alpha)} B(1-\sigma, \alpha)+\frac{t^{\alpha-\sigma}-t_{0}^{\alpha-\sigma}}{\Gamma(\alpha)} B(1-\sigma, \alpha)+\frac{a\left[t^{\alpha-1}-t_{0}^{\alpha-1}\right]}{\Gamma\left(\frac{\alpha+1}{2}\right)-a \Gamma(\alpha) \xi^{\frac{\alpha-1}{2}}}\right. \\
& \left.\times\left[\xi^{\frac{\alpha-1}{2}} B(1-\sigma, \alpha)+\xi^{1-\sigma+\frac{\alpha-1}{2}} B\left(1-\sigma, \frac{\alpha-1}{2}\right)\right]\right\} \\
& \rightarrow 0 \quad\left(t \rightarrow t_{0}\right) \text {. }
\end{aligned}
$$

Case 3. $t_{0} \in(0,1], \forall t \in\left[0, t_{0}\right)$. The proof is similar to that of Case 2 , so we omit it.

Let Banach space $E=C[0,1]$ be endowed with the norm $\|u\|=\max _{t \in[0,1]}|u(t)|$. Note that this space can be equipped with a partial order given by

$$
x, y \in E, \quad x \leq y \quad \Leftrightarrow \quad x(t) \leq y(t), \quad t \in[0,1] .
$$

It is easy to check that $(E, \leq)$ with the classic metric given by

$$
d(x, y)=\max _{t \in[0,1]}\{|x(t)-y(t)|\}
$$

satisfies condition (2.6) of Lemma 2.5. Moreover, for $x, y \in E$, as the function $\max \{x, y\}$ is continuous in $[0,1],(E, \leq)$ satisfies condition $(2.7)$.

Theorem 3.2 Let $0<\sigma<1,1<\alpha \leq 2, \xi \in\left(0, \frac{1}{2}\right], a \in(0,+\infty)$ satisfy that $a \Gamma(\alpha) \xi^{\frac{\alpha-1}{2}}<$ $\Gamma\left(\frac{\alpha+1}{2}\right), f:(0,1] \times[0,+\infty) \rightarrow[0,+\infty)$ is continuous, with $\lim _{t \rightarrow 0^{+}} f(t, \cdot)=+\infty$, and $t^{\sigma} f(t, u)$ is continuous function on $[0,1] \times[0,+\infty)$. Assume that there exists $\lambda$ satisfying

$$
\begin{aligned}
0<\lambda & \\
\leq & {\left[\frac{\Gamma(1-\sigma)}{\Gamma(1-\sigma+\alpha)}+\frac{\Gamma\left(\frac{\alpha+1}{2}\right) \Gamma(1-\sigma)}{\left[\Gamma\left(\frac{\alpha+1}{2}\right)-a \Gamma(\alpha) \xi^{\frac{\alpha-1}{2}}\right] \Gamma(1-\sigma+\alpha)}\right.} \\
& \left.+\frac{a \xi^{\left(1-\sigma+\frac{\alpha-1}{2}\right)} \Gamma(1-\sigma) \Gamma\left(\frac{\alpha+1}{2}\right)}{\left[\Gamma\left(\frac{\alpha+1}{2}\right)-a \Gamma(\alpha) \xi^{\frac{\alpha-1}{2}}\right] \Gamma\left(1-\sigma+\frac{\alpha+1}{2}\right)}\right]^{-1},
\end{aligned}
$$


such that for $u, v \in[0,+\infty)$ with $u \geq v$ and $t \in[0,1]$

$$
0 \leq t^{\sigma}[f(t, u)-f(t, v)] \leq \lambda \phi(u-v)
$$

where $\phi:[0,+\infty) \rightarrow[0,+\infty)$ is continuous and nondecreasing, $\varphi(u)=u-\phi(u)$ satisfies

(a) $\varphi:[0,+\infty) \rightarrow[0,+\infty)$ and nondecreasing;

(b) $\varphi(0)=0$;

(c) $\varphi$ is positive in $(0,+\infty)$.

Then the problem (1.1)-(1.2) has an unique positive solution.

Proof Define the cone $\mathcal{K} \subset E$ by

$$
\mathcal{K}=\{u \in E: u(t) \geq 0, t \in[0,1]\}
$$

Note that, as $\mathcal{K}$ is a closed subset of $E, \mathcal{K}$ is a complete metric space.

Suppose that $u$ is a solution of boundary value problem (1.1) and (1.2). Then

$$
\begin{aligned}
u(t)= & \int_{0}^{1} G(t, s) f(s, u(s)) d s+\frac{a t^{\alpha-1}}{\Gamma\left(\frac{\alpha+1}{2}\right)-a \Gamma(\alpha) \xi^{\frac{\alpha-1}{2}}} \\
& \times\left\{\int_{0}^{\xi}\left[(1-s)^{\alpha-1} \xi^{\frac{\alpha-1}{2}}-(\xi-s)^{\frac{\alpha-1}{2}}\right] f(s, u(s)) d s\right. \\
& \left.+\int_{\xi}^{1}(1-s)^{\alpha-1} \xi^{\frac{\alpha-1}{2}} f(s, u(s)) d s\right\}, \quad t \in[0,1] .
\end{aligned}
$$

Define an operator $\mathcal{A}: \mathcal{K} \rightarrow E$ as follows:

$$
\begin{aligned}
(\mathcal{A} u)(t)= & \int_{0}^{1} G(t, s) f(s, u(s)) d s+\frac{a t^{\alpha-1}}{\Gamma\left(\frac{\alpha+1}{2}\right)-a \Gamma(\alpha) \xi^{\frac{\alpha-1}{2}}} \\
& \times\left\{\int_{0}^{\xi}\left[(1-s)^{\alpha-1} \xi^{\frac{\alpha-1}{2}}-(\xi-s)^{\frac{\alpha-1}{2}}\right] f(s, u(s)) d s\right. \\
& \left.+\int_{\xi}^{1}(1-s)^{\alpha-1} \xi^{\frac{\alpha-1}{2}} f(s, u(s)) d s\right\}, \quad t \in[0,1] .
\end{aligned}
$$

By Theorem 3.1, $\mathcal{A} u \in E$. Moreover, in view of Lemma 2.4 and $t^{\sigma} f(t, u) \geq 0$ for $(t, u) \in$ $[0,1] \times[0,+\infty)$, by hypothesis, we get

$$
(\mathcal{A} u)(t) \geq 0, \quad t \in[0,1]
$$

so, $\mathcal{A}(\mathcal{K}) \subset \mathcal{K}$.

In what follows, we check that hypotheses in Lemmas 2.5 and 2.6 are satisfied. Firstly, the operator $\mathcal{A}$ is nondecreasing. By hypothesis, for $u \geq v$, we get

$$
\begin{aligned}
(\mathcal{A} u)(t)= & \int_{0}^{1} G(t, s) f(s, u(s)) d s+\frac{a t^{\alpha-1}}{\Gamma\left(\frac{\alpha+1}{2}\right)-a \Gamma(\alpha) \xi^{\frac{\alpha-1}{2}}} \\
& \times\left\{\int_{0}^{\xi}\left[(1-s)^{\alpha-1} \xi^{\frac{\alpha-1}{2}}-(\xi-s)^{\frac{\alpha-1}{2}}\right] f(s, u(s)) d s\right.
\end{aligned}
$$




$$
\begin{aligned}
& \left.+\int_{\xi}^{1}(1-s)^{\alpha-1} \xi^{\frac{\alpha-1}{2}} f(s, u(s)) d s\right\} \\
\geq & \int_{0}^{1} G(t, s) f(s, v(s)) d s+\frac{a t^{\alpha-1}}{\Gamma\left(\frac{\alpha+1}{2}\right)-a \Gamma(\alpha) \xi^{\frac{\alpha-1}{2}}} \\
& \times\left\{\int_{0}^{\xi}\left[(1-s)^{\alpha-1} \xi^{\frac{\alpha-1}{2}}-(\xi-s)^{\frac{\alpha-1}{2}}\right] f(s, v(s)) d s\right. \\
& \left.+\int_{\xi}^{1}(1-s)^{\alpha-1} \xi^{\frac{\alpha-1}{2}} f(s, v(s)) d s\right\} \\
= & (\mathcal{A} v)(t) .
\end{aligned}
$$

Besides, for $u \geq v$, by (3.4), we get

$$
\begin{aligned}
d(\mathcal{A} u, \mathcal{A} v)= & \max _{t \in[0,1]}\{|\mathcal{A} u(t)-\mathcal{A} v(t)|\} \\
= & \max _{t \in[0,1]}[\mathcal{A} u(t)-\mathcal{A} v(t)] \\
\leq & \max _{t \in[0,1]}\left[\int_{0}^{1} G(t, s)[f(s, u(s))-f(s, v(s))] d s+\frac{a t^{\alpha-1}}{\Gamma\left(\frac{\alpha+1}{2}\right)-a \Gamma(\alpha) \xi^{\frac{\alpha-1}{2}}}\right. \\
& \times\left\{\int_{0}^{\xi}\left[(1-s)^{\alpha-1} \xi^{\frac{\alpha-1}{2}}-(\xi-s)^{\frac{\alpha-1}{2}}\right][f(s, u(s))-f(s, v(s))] d s\right. \\
& \left.\left.+\int_{\xi}^{1}(1-s)^{\alpha-1} \xi^{\frac{\alpha-1}{2}}[f(s, u(s))-f(s, v(s))] d s\right\}\right] \\
\leq & \max _{t \in[0,1]}\left[\int_{0}^{1} G(t, s) s^{-\sigma} \lambda \phi[u(s)-v(s)] d s+\frac{a t^{\alpha-1}}{\Gamma\left(\frac{\alpha+1}{2}\right)-a \Gamma(\alpha) \xi^{\frac{\alpha-1}{2}}}\right. \\
& \times\left\{\int_{0}^{\xi}\left[(1-s)^{\alpha-1} \xi^{\frac{\alpha-1}{2}}-(\xi-s)^{\frac{\alpha-1}{2}}\right] s^{-\sigma} \lambda \phi[u(s)-v(s)] d s\right. \\
& \left.\left.+\int_{\xi}^{1}(1-s)^{\alpha-1} \xi^{\frac{\alpha-1}{2}} s^{-\sigma} \lambda \phi[u(s)-v(s)] d s\right\}\right] .
\end{aligned}
$$

As the function $\phi(u)$ is nondecreasing, for $u \geq v$, we get

$$
\phi[u(s)-v(s)] \leq \phi(\|u-v\|) .
$$

By the last inequality, we get

$$
\begin{aligned}
d(\mathcal{A} u, \mathcal{A} v) \leq & \max _{t \in[0,1]}\left[\int_{0}^{1} G(t, s) s^{-\sigma} \lambda \phi[u(s)-v(s)] d s+\frac{a t^{\alpha-1}}{\Gamma\left(\frac{\alpha+1}{2}\right)-a \Gamma(\alpha) \xi^{\frac{\alpha-1}{2}}}\right. \\
& \times\left\{\int_{0}^{\xi}\left[(1-s)^{\alpha-1} \xi^{\frac{\alpha-1}{2}}-(\xi-s)^{\frac{\alpha-1}{2}}\right] s^{-\sigma} \lambda \phi[u(s)-v(s)] d s\right. \\
& \left.\left.+\int_{\xi}^{1}(1-s)^{\alpha-1} \xi^{\frac{\alpha-1}{2}} s^{-\sigma} \lambda \phi[u(s)-v(s)] d s\right\}\right] \\
\leq & \lambda \phi(\|u-v\|) \max _{t \in[0,1]}\left[\int_{0}^{1} G(t, s) s^{-\sigma} d s+\frac{a t^{\alpha-1}}{\Gamma\left(\frac{\alpha+1}{2}\right)-a \Gamma(\alpha) \xi^{\frac{\alpha-1}{2}}}\right.
\end{aligned}
$$




$$
\begin{aligned}
& \left.\times\left\{\int_{0}^{\xi}\left[(1-s)^{\alpha-1} \xi^{\frac{\alpha-1}{2}}-(\xi-s)^{\frac{\alpha-1}{2}}\right] s^{-\sigma} d s+\int_{\xi}^{1}(1-s)^{\alpha-1} \xi^{\frac{\alpha-1}{2}} s^{-\sigma} d s\right\}\right] \\
= & \lambda \phi(\|u-v\|) \max _{t \in[0,1]}\left[\frac{t^{\alpha-\sigma}}{\Gamma(\alpha)} B(1-\sigma, \alpha)+\frac{t^{\alpha-1} \Gamma\left(\frac{\alpha+1}{2}\right) B(1-\sigma, \alpha)}{\left[\Gamma\left(\frac{\alpha+1}{2}\right)-a \Gamma(\alpha) \xi^{\frac{\alpha-1}{2}}\right] \Gamma(\alpha)}\right. \\
& \left.+\frac{a t^{\alpha-1} \xi^{\left(1-\sigma+\frac{\alpha-1}{2}\right)}}{\left[\Gamma\left(\frac{\alpha+1}{2}\right)-a \Gamma(\alpha) \xi^{\frac{\alpha-1}{2}}\right]} B\left(1-\sigma, \frac{\alpha+1}{2}\right)\right] \\
= & \lambda \phi(\|u-v\|)\left[\frac{B(1-\sigma, \alpha)}{\Gamma(\alpha)}+\frac{\Gamma\left(\frac{\alpha+1}{2}\right) B(1-\sigma, \alpha)}{\left[\Gamma\left(\frac{\alpha+1}{2}\right)-a \Gamma(\alpha) \xi^{\frac{\alpha-1}{2}}\right] \Gamma(\alpha)}\right. \\
& \left.+\frac{a \xi^{\left(1-\sigma+\frac{\alpha-1}{2}\right)}}{\left[\Gamma\left(\frac{\alpha+1}{2}\right)-a \Gamma(\alpha) \xi^{\frac{\alpha-1}{2}}\right]} B\left(1-\sigma, \frac{\alpha+1}{2}\right)\right] \\
= & \lambda \phi(\|u-v\|)\left[\frac{\Gamma(1-\sigma)}{\Gamma(1-\sigma+\alpha)}+\frac{\Gamma\left(\frac{\alpha+1}{2}\right) \Gamma(1-\sigma)}{\left[\Gamma\left(\frac{\alpha+1}{2}\right)-a \Gamma(\alpha) \xi^{\frac{\alpha-1}{2}}\right] \Gamma(1-\sigma+\alpha)}\right. \\
& +\frac{a \xi\left(1-\sigma+\frac{\alpha-1}{2}\right)}{\left[\Gamma(1-\sigma) \Gamma\left(\frac{\alpha+1}{2}\right)\right.} \\
\leq & \phi(\|u-v\|) \\
= & \|u-v\|-[\|u-v\|-\phi(\|u-v\|)] .
\end{aligned}
$$

Put $\varphi(u)=u-\phi(u)$. Obviously, $\varphi:[0,+\infty) \rightarrow[0,+\infty)$ is continuous, nondecreasing, positive in $(0,+\infty), \varphi(0)=0$.

Thus, for $u \geq v$, we get

$$
d(\mathcal{A} u, \mathcal{A} v) \leq d(u, v)-\varphi(d(u, v)) .
$$

Finally, take into account that for the zero function, $0 \leq \mathcal{A} 0$, by Lemma 2.5 , our problem (1.1)-(1.2) has at least one nonnegative solution. Moreover, this solution is unique, since $(\mathcal{K}, \leq)$ satisfies condition (2.7) and Lemma 2.6. This completes the proof.

In the sequel, we present an example which illustrates Theorem 3.2.

\section{An example}

Example 4.1 Consider the following fractional boundary value problem:

$$
\begin{aligned}
& D^{\frac{3}{2}} u(t)=\frac{\left(t-\frac{1}{2}\right)^{2} \ln (2+u(t))}{\sqrt{t}}, \quad 0<t<1, \\
& u(0)=0, \quad u(1)=\left.\frac{1}{2} D^{\frac{1}{4}} u(t)\right|_{t=\frac{1}{2}},
\end{aligned}
$$

where $\alpha=\frac{3}{2}, a=\xi=\frac{1}{2}$. In this case, $f(t, u)=\frac{\left(t-\frac{1}{2}\right)^{2} \ln (2+u)}{\sqrt{t}}$ for $(t, u) \in(0,1] \times[0,+\infty), \sigma=\frac{1}{2}$. Note that $f$ is continuous in $(0,1] \times[0,+\infty)$ and $\lim _{t \rightarrow 0^{+}} f(t, \cdot)=\infty$. Moreover, for $u \geq v$ and $t \in[0,1]$, we have

$$
0 \leq t^{\sigma}[f(t, u)-f(t, v)]=\left[\left(t-\frac{1}{2}\right)^{2} \ln (2+u)-\left(t-\frac{1}{2}\right)^{2} \ln (2+v)\right]
$$


Because $g(x)=\ln (x+2)$ is nondecreasing on $[0,+\infty)$, and

$$
\begin{aligned}
{\left[\left(t-\frac{1}{2}\right)^{2} \ln (2+u)-\left(t-\frac{1}{2}\right)^{2} \ln (2+v)\right] } & =\left(t-\frac{1}{2}\right)^{2} \ln \frac{(2+u)}{(2+v)} \\
& =\left(t-\frac{1}{2}\right)^{2} \ln \frac{(2+v+u-v)}{(2+v)} \\
& \leq\left(\frac{1}{2}\right)^{2} \ln (1+u-v)
\end{aligned}
$$

With the aid of a computer, we obtain that

$$
\begin{aligned}
& {\left[\frac{\Gamma(1-\sigma)}{\Gamma(1-\sigma+\alpha)}+\frac{\Gamma\left(\frac{\alpha+1}{2}\right) \Gamma(1-\sigma)}{\left[\Gamma\left(\frac{\alpha+1}{2}\right)-a \Gamma(\alpha) \xi^{\frac{\alpha-1}{2}}\right] \Gamma(1-\sigma+\alpha)}\right.} \\
& \left.\quad+\frac{a \xi^{\left(1-\sigma+\frac{\alpha-1}{2}\right)} \Gamma(1-\sigma) \Gamma\left(\frac{\alpha+1}{2}\right)}{\left[\Gamma\left(\frac{\alpha+1}{2}\right)-a \Gamma(\alpha) \xi^{\frac{\alpha-1}{2}}\right] \Gamma\left(1-\sigma+\frac{\alpha+1}{2}\right)}\right]^{-1} \approx 0.282 \cdots>\frac{1}{4}
\end{aligned}
$$

So, by Theorem 3.2, the problem (4.1)-(4.2) has an unique positive solution.

\section{Competing interests}

The authors declare that they have no competing interests.

\section{Authors' contributions}

All authors contributed equally to the manuscript. All authors read and approved the final manuscript.

\section{Author details}

${ }^{1}$ School of Mathematical Sciences, Fudan University, Shanghai, 200433, China. ${ }^{2}$ Department of Mathematics, Lanzhou Jiaotong University, Lanzhou, 730070, China. ${ }^{3}$ Department of Mathematics and Computer Science, Çankaya University, 06530, Balgat, Ankara, Turkey. ${ }^{4}$ Institute of Space Sciences, P.O. Box MG-23, Magurele, Bucharest 76900, Romania.

\section{Acknowledgements}

Dedicated to Professor Hari M Srivastava.

The authors are thankful to the referees for their careful reading of the manuscript and insightful comments. The research is supported by the National Natural Science Foundation of China (11161027, 11262009).

\section{Received: 10 December 2012 Accepted: 8 April 2013 Published: 19 April 2013}

\section{References}

1. Kilbas, AA, Srivastava, HM, Trujillo, JJ: Theory and Applications of Fractional Differential Equations. North-Holland Mathematics Studies, vol. 204. Elsevier, Amsterdam (2006)

2. Miller, KS, Ross, B: An Introduction to the Fractional Calculus and Differential Equations. Wiley, New York (1993)

3. Oldham, KB, Spanier, J: The Fractional Calculus. Academic Press, New York (1974)

4. Podlubny, I: Fractional Differential Equation. Mathematics in Science and Engineering. Academic Press, New York (1999)

5. Samko, SG, Kilbas, AA, Igorevich, O: Fractional Integrals and Derivatives: Theory and Applications. Gordon \& Breach, Yverdon (1993)

6. Lakshmikantham, V, Vatsala, AS: Basic theory of fractional differential equations. Nonlinear Anal. 69, 2677-2682 (2008)

7. Agarwal, RP, Zhou, Y, Wang, JR, Luo, XN: Fractional functional differential equations with causal operators in Banach spaces. Math. Comput. Model. 54, 1440-1452 (2011)

8. Chang, Y, Nieto, J: Some new existence results for fractional differential inclusions with boundary conditions. Math. Comput. Model. 49, 605-609 (2009)

9. Li, CF, Luo, XN, Zhou, Y: Existence of positive solutions of the boundary value problem for nonlinear fractional differential equations. Comput. Math. Appl. 59, 1363-1375 (2010)

10. Zhou, Y, Jiao, F, Li, J: Existence and uniqueness for fractional neutral differential equations with infinite delay. Nonlinear Anal. 71, 3249-3256 (2009)

11. Zhou, Y: Existence and uniqueness of solutions for a system of fractional differential equations. Fract. Calc. Appl. Anal. 12, 195-204 (2009)

12. Jiao, F, Zhou, Y: Existence of solutions for a class of fractional boundary value problems via critical point theory. Comput. Math. Appl. 62, 1181-1199 (2011) 
13. Wang, JR, Zhou, Y: Analysis of nonlinear fractional control systems in Banach spaces. Nonlinear Anal. 74, 5929-5942 (2011)

14. Wang, GT, Ahmad, B, Zhang, LH: Impulsive anti-periodic boundary value problem for nonlinear differential equations of fractional order. Nonlinear Anal. 74, 792-804 (2011)

15. Wang, GT, Ahmad, B, Zhang, LH: Some existence results for impulsive nonlinear fractional differential equations with mixed boundary conditions. Comput. Math. Appl. 62, 1389-1397 (2011)

16. Zhou, WX, Chu, YD: Existence of solutions for fractional differential equations with multi-point boundary conditions. Commun. Nonlinear Sci. Numer. Simul. 17, 1142-1148 (2012)

17. Delbosco, D, Rodino, L: Existence and uniqueness for a nonlinear fractional differential equation. J. Math. Anal. Appl. 204, 609-625 (1996)

18. Qiu, T, Bai, Z: Existence of positive solutions for singular fractional differential equations. Electron. J. Differ. Equ. 2008 $146(2008)$

19. Kosmatov, N: A singular boundary value problem for nonlinear differential equations of fractional order. J. Appl. Math Comput. 29, 125-135 (2009)

20. Caballero Mena, J, Harjani, J, Sadarangani, K: Existence and uniqueness of positive and nondecreasing solutions for a class of singular fractional boundary value problems. Bound. Value Probl. 2009, Article ID 421310 (2009)

21. Tian, $Y, C$ Chen, $A$ : The existence of positive solution to three-point singular boundary value problem of fractional differential equation. Abstr. Appl. Anal. 2009, Article ID 314656 (2009)

22. Xu, X, Jiang, D, Yuan, C: Multiple positive solutions for the boundary value problem of a nonlinear fractional differential equation. Nonlinear Anal. 71, 4676-4688 (2009)

23. Zhang, S: Nonnegative solution for singular nonlinear fractional differential equation with coefficient that changes sign. Positivity 12, 711-724 (2008)

24. Harjani, J, Sadarangani, K: Fixed point theorems for weakly contractive mappings in partially ordered sets. Nonlinear Anal. 71, 3403-3410 (2009)

doi:10.1186/1687-1847-2013-114

Cite this article as: Zhou et al.: Uniqueness and existence of positive solutions for a multi-point boundary value problem of singular fractional differential equations. Advances in Difference Equations 2013 2013:114.

\section{Submit your manuscript to a SpringerOpen ${ }^{\ominus}$ journal and benefit from:}

- Convenient online submission

- Rigorous peer review

- Immediate publication on acceptance

- Open access: articles freely available online

- High visibility within the field

- Retaining the copyright to your article 